\title{
Nuevos registros de Phthiraptera (Artropoda: Insecta) en Lama pacos Linnaeus 1758, en Chile
}

\author{
New records of Phthiraptera (Artropoda: Insecta) in Lama pacos Linnaeus 1758, in Chile \\ D González-Acuña ${ }^{1 *}$, I Cabezas ${ }^{1}$, L Moreno ${ }^{1}$, D Castro ${ }^{2}$ \\ ${ }^{1}$ Facultad de Medicina Veterinaria, Universidad de Concepción, Chillán, Chile. \\ ${ }^{2}$ Facultad de Ciencias Naturales y Museo de La Plata, Lab. 32, calle 64 № 3, 1900, La Plata, Buenos Aires, Argentina.
}

\begin{abstract}
SUMMARY
For the first time in Chile, the presence of Microthoracius mazzai (Werneck 1932) and Bovicola breviceps (Rudow 1866), are reported, and the presence of M. praelongiceps (Neuman 1909) parasiting alpacas in the General Lagos area (I Region) is also confirmed. The importance of this situation is discussed in the present report.
\end{abstract}

Palabras clave: alpacas, Microthoracius, Bovicola, Chile.

Key words: alpacas, Microthoracius, Bovicola, Chile.

\section{INTRODUCCION}

La alpaca (Lama pacos Linnaeus 1758) es uno de los dos camélidos sudamericanos (CSA) domesticados por el hombre. Se distribuye en tierras altas de la cordillera de los Andes, a una altura de 4000 metros, principalmente desde el norte del Perú a la región norte de Chile y Bolivia (MacDonald 2001). En Chile, la mayor parte de la población se encuentra en el altiplano de la región de Tarapacá (I Región) (Bas y González 1990), alcanzando un total de 45.282 animales (INE 1997).

Dadas las condiciones de producción extensiva y semiintensiva de la alpaca, el parasitismo se ha transformado en una problemática de alta trascendencia económica, ya que constituye el principal problema sanitario en su explotación (Leguía 1991). Los parásitos pueden producir cuadros estresantes que conducen a una modificación sustancial tanto de comportamiento como de eventual desarrollo productivo (Castro y Cicchino 1998), además, estos cuadros pueden ser agravados por la presencia de microorganismos oportunistas, lo que se traduce en una merma de ingresos para el productor (Leguía 1991).

Los Phthiraptera en CSA domésticos (alpacas y llamas) han sido estudiados por Neumann (1909), Fahrenholz (1916), Werneck (1932, 1933), Ferris (1951), Büttiker (1967) y Castro y Cicchino (1987). En Argentina han sido registrados en alpacas Bovicola breviceps (Rudow 1866), Microthoracius praelongiceps (Neumann 1909) у M.

Aceptado: 11.04.2006.

Facultad de Medicina Veterinaria, Universidad de Concepción, Casilla 537, Fax: 42-270212, Chillán, Chile.

E-mail: danigonz@udec.cl minor Werneck 1935 (Werneck 1936, Castro y Cicchino 1987) y en Perú M. mazzai Werneck 1932 (Büttiker 1967). En Chile, en CSA domésticos ha sido registrado hasta ahora sólo el Phthiraptera M. praelongiceps (Alcaíno y Gorman 1999). En la presente nota citamos por primera vez a $M$. mazzai y B. breviceps en alpacas en Chile.

\section{MATERIAL Y METODOS}

Durante el mes de febrero de 2004 fueron muestreadas un total de 59 alpacas en tres sectores (Visviri $17^{\circ} 35^{\prime} \mathrm{S}$ $69^{\circ} 28^{\prime} \mathrm{O}, 4095$ m.s.n.m; Tacora a $35 \mathrm{~km}$ al suroeste de Visviri, 4500 m.s.n.m y Humaquilca ubicado a $29 \mathrm{~km}$ sureste de Visviri, 4300 m.s.n.m) pertenecientes a la comuna de General Lagos, I Región, Chile.

Los Phthiraptera fueron colectados en forma manual y conservados en alcohol 70\%, posteriormente lavados en $\mathrm{KOH}$ al 20\% y luego sometidos a soluciones ascendentes de alcohol $(40 \%, 80 \%$ y $100 \%)$. Se aclararon durante 24 horas en aceite de clavo, montados en Bálsamo de Canadá (Palma 1978) para luego ser identificados siguiendo las pautas de Neumann (1909), Werneck (1932, 1933, 1935) y Ferris (1951). El material estudiado fue depositado en la colección del laboratorio de Zoología de la Facultad de Medicina Veterinaria de la Universidad de Concepción.

\section{RESULTADOS Y DISCUSION}

Del total de alpacas estudiadas ( $\mathrm{n}=59)$, el $40,7 \%$ $(n=24)$, resultó positivo a la presencia de Phthiraptera. Fueron colectados un total de 42 especímenes, de los cuales 35 correspondieron a $M$. praelongiceps, cinco a M. mazzai y dos a B. breviceps (cuadro 1). 
Cuadro 1. Phthiraptera colectados en alpacas en tres sectores pertenecientes a la comuna de General Lagos, I Región, Chile.

Phthiraptera collected from alpacas in three different areas of General Lagos area, I Region, Chile.

\begin{tabular}{lccccc}
\hline $\begin{array}{l}\text { Phthiraptera } \\
\text { colectados }\end{array}$ & Sector & $\begin{array}{c}\mathrm{N}^{\circ} \\
\text { colectado }\end{array}$ & + & $\hat{0}$ & $\mathrm{~N}$ \\
\hline $\begin{array}{l}\text { M. praelongiceps } \\
\text { M. mazzai }\end{array}$ & $1,2,3$ & 35 & 12 & 23 & - \\
B. breviceps & 3 & 5 & 1 & 4 & - \\
\hline Total & 2 & 2 & - & - & 2 \\
\hline
\end{tabular}

1: Visviri 2: Tacora 3: Humaquilca $\quad \mathrm{N}$ : ninfa

El género Microthoracius Fahrenholz 1916 comprende cuatro especies: M. praelongiceps, M. mazzai, M. minor y M. cameli, todas restringidas a individuos de la familia Camelidae (Castro y Cicchino 1998).

M. mazzai fue descrito por primera vez por Neumann en 1909 aislado de Lama guanicoe (Müller 1776) en Choquecomato, Bolivia. En esta descripción Neumann confunde las especies praelongiceps y mazzai describiéndolas como una sola y dándole el nombre de Haematopinus praelongiceps. Luego Werneck en 1932 analiza muestras de L. glama (Linnaeus 1758) provenientes de Santa Catalina, Provincia de Jujuy, Argentina, y las compara con las analizadas por Neumann, verificando que se trataba de dos especies y que por diversas circunstancias habían sido confundidas. Werneck (1932) conserva el nombre de praelongiceps para una de las especies y a la otra le da el nombre de mazzai. En 1933 Werneck presenta otro trabajo donde describe las diferencias entre estas dos especies y los motivos que llevaron a su confusión. Ferris (1951) describe esta especie en llamas criadas en el Jardín Zoológico de Washington, D.F., EE.UU. Más tarde Büttiker (1967) aísla M. mazzai desde alpacas en Sarapata, Puno, Perú. Esta especie también ha sido citada en Argentina parasitando a L. pacos, L. guanicoe y L. glama (Castro y Cicchino 1998).

$M$. praelongiceps ha sido registrada en $L$. glama, en el Zoological Garden at Onderstepoort, Sudáfrica (Ferris 1951), y en Argentina en L. pacos, L. guanicoe y L. glama (Castro y Cicchino 1998). En Chile, M. praelongiceps fue registrado por Alcaíno y Gorman (1999), no especificando el lugar de colección e indicando como hospedador a llama y alpaca.

En Chile, hasta la fecha, no se ha citado a M. minor parasitando alpacas, aunque su presencia es muy probable, ya que ha sido citada para países limítrofes sobre este hospedador (Werneck 1935, Castro y Cicchino 1998); seguramente una revisión más exhaustiva de estos camélidos corroborará su presencia en Chile.

Bovicola es un género cosmopolita, posee un total de 15 especies en cuatro subgéneros, parasita artyodactilos de las familias Bovidae, Cervidae y Camelidae (Cicchino y Castro 1998). B. breviceps ha sido descrito en L. glama en Pachacayo, Perú, L. guanicoe y L. pacos en Abra Pampa, Argentina (Werneck 1950).

Las presentes nuevas citas en Chile son de importancia debido a que $B$. breviceps ha sido, por Cicchino y Castro (1998), incriminada como una especie que provoca alteraciones en el comportamiento de sus hospedadores causando deterioro en la performance productiva. Por otro lado, la especie M. mazzai ha sido catalogada como el piojo más importante en la explotación intensiva de camélidos sudamericanos, el cual produce una baja importante en la ganancia de peso, debido al estrés y alteración de la fibra por el excesivo rascado (Castro y Cicchino 1998).

\section{RESUMEN}

Se cita a Microthoracius mazzai (Werneck 1932) y Bovicola breviceps (Rudow 1866) por primera vez en Chile, y se confirma la presencia de M. praelongiceps (Neumann 1909) parasitando alpacas en la comuna de General Lagos (I Región), Chile. Se discute la importancia de los presentes registros.

\section{REFERENCIAS}

Alcaíno H, T Gorman. 1999. Parásitos de animales domésticos en Chile. Parasitol día 23, 1-2.

Bas F, F González. 1990. Antecedentes para la producción de alpacas en la zona central de Chile. Panorama económico de la agricultura 75, 23-27.

Büttiker W. 1967. Nova descoberta de Microthoracius mazzai Werneck (Haematopinidea, Anoplura) na Alpaca (Lama pacos L.). Rev Bras Entomol 12, 159-163.

Castro D del C, A Cicchino. 1987. Lista referencial de los Anoplura y Mallophaga (Insecta) conocidos como parásitos de mamíferos en la Argentina. Rev Soc Entomol Argentina 44, 357-370.

Castro D del C, AC Cicchino. 1998. Anoplura. En: Morrone JJ, Coscaron S (eds.). Biodiversidad de artrópodos argentinos. Ediciones Sur. La Plata, Argentina, Pp 125-139.

Cicchino AC, D del C Castro. 1998. Ischnocera, En: Morrone JJ, Coscaron S (eds.). Biodiversidad de artrópodos argentinos. Ediciones Sur. La Plata, Argentina, Pp 104-123.

Fahrenholz H. 1916. Weitere Beiträge zur Kenntnis der Anopluren. Archiv für Naturgeschichte 81 A, 1-34.

Ferris GF. 1951. The sucking lice. Mem Pacific Coast Entomol Society, Volumen 1.

INE Chile. 1997. V Censo Nacional Agropecuario. 1975-1976. Instituto Nacional de Estadísticas (INE), Santiago, Chile, Pp 2657.

Leguía G. 1991. The epidemiology and economic impact of llama parasites. Parasitol Today 7, 54-56.

MacDonald D. 2001. The new encyclopedia of mammals. Oxford Press. Italy.

Neumann LG. 1909. Notes sur les Pédiculidés. Arch Parasitol 13, $497-$ 537.

Palma R. 1978. Slid- mounting of lice: a detailed description of the Canada balsam technique. $N$ Z Entomol 6, 432-436.

Werneck F. 1932. Sobre uma nova especie de Anoplura parasita da lhama. Rev med-cirurg Brasil 40, 346-348.

Werneck F. 1933. Sobre as especies de Anoplura parasitas da lhama. Memorias del Instituto Oswaldo Cruz 27, 21-32.

Werneck F. 1935. Microthoracius minor e demais especies do mesmo genero. Rev Ent Rio de J 5, 107-116.

Werneck, F. 1936. Contribuição ao conhecimento dos Mallophagos encontrados nos mammiferos sul-americanos. Memorias del Instituto Oswaldo Cruz 31, 391-87.

Werneck, F. 1950. Os malófagos de mamíferos. Parte II: Ischnocera. (continuación de Trichodectidae) e Rhyncophthirina. Memorias del Instituto Oswaldo Cruz, Pp 1-207. 